\title{
PUTNAM-FUGLEDE THEOREM AND THE RANGE-KERNEL ORTHOGONALITY OF DERIVATIONS
}

\author{
B. P. DUGGAL
}

(Received 3 November 2000)

\begin{abstract}
Let $\mathscr{B}(H)$ denote the algebra of operators on a Hilbert space $H$ into itself. Let $d=\delta$ or $\triangle$, where $\delta_{A B}: \mathscr{B}(H) \rightarrow \mathscr{B}(H)$ is the generalized derivation $\delta_{A B}(S)=A S-S B$ and $\triangle_{A B}: \mathscr{B}(H) \rightarrow \mathscr{B}(H)$ is the elementary operator $\triangle_{A B}(S)=A S B-S$. Given $A, B, S \in \mathscr{B}(H)$, we say that the pair $(A, B)$ has the property $\operatorname{PF}(d(S))$ if $d_{A B}(S)=0$ implies $d_{A^{*} B^{*}}(S)=$ 0 . This paper characterizes operators $A, B$, and $S$ for which the pair $(A, B)$ has property $\operatorname{PF}(d(S))$, and establishes a relationship between the $\operatorname{PF}(d(S))$-property of the pair $(A, B)$ and the range-kernel orthogonality of the operator $d_{A B}$.
\end{abstract}

2000 Mathematics Subject Classification. 47A62, 47B47, 47B10.

1. Introduction. Let $H$ be a complex Hilbert space, and let $\mathscr{B}(H)$ denote the algebra of operators (i.e., bounded linear transformations) on $H$ into itself. Given $A, B \in \mathscr{B}(H)$, the (classical) Putnam-Fuglede commutativity theorem says that if $A, B$ are normal operators, and if $X$ is an operator such that $A X=X B$, then $A^{*} X=X B^{*}$ [9, page 104]. Various generalizations of the Putnam-Fuglede theorem (henceforth shortened to PFtheorem) have appeared over the past three decades (see $[4,8,13,14,15,17]$ and some of the references cited in these papers). A generalization of the PF-theorem is obtained when the normality of $A$ and $B$ is replaced by a weaker requirement, such as $A$ and $B^{*}$ are subnormal or hyponormal. (Here the hypotheses on $A, B$ are asymmetric: there exist subnormal operators $A$ and $B$, and operators $X$, such that $A X=X B$ but $A^{*} X \neq X B^{*}$ [9, Problem 199, page 107].) Another such generalization of the PF-theorem, considered recently by Okuyama and Watanabe [14], is where the requirement that $A$ and $B$ be normal is removed by requiring more of the intertwining operator $X$.

Let $\delta_{A B}: \mathscr{B}(H) \rightarrow \mathscr{B}(H)\left(\delta_{A A}=\delta_{A}\right)$ denote the generalized derivation $\delta_{A B}(X)=$ $A X-X B$, and let $\triangle_{A B}: \mathscr{B}(H) \rightarrow \mathscr{B}(H)\left(\triangle_{A A}=\triangle_{A}\right)$ denote the elementary operator $\triangle_{A B}(X)=A X B-X$. Let $\operatorname{ker}(Y)$ denote the kernel of the operator $Y$. The (classical) PF-theorem then says that if $A, B$ are normal, then $\operatorname{ker}\left(\delta_{A B}\right)=\operatorname{ker}\left(\delta_{A^{*} B^{*}}\right)$. There is a natural $\triangle_{A B}$ analogue, namely that if $A, B$ are normal, then $\operatorname{ker}\left(\triangle_{A B}\right)=\operatorname{ker}\left(\triangle_{A^{*} B^{*}}\right)$. Let $d$ denote either $\delta$ or $\triangle$. We say that the pair of operators $(A, B)$ has the property $\operatorname{PF}(d)$ (the property $\operatorname{PF}(d(S)))$ if $\operatorname{ker}\left(d_{A B}\right) \subseteq \operatorname{ker}\left(d_{A^{*} B^{*}}\right)$ (resp., if, given $S \in \mathscr{B}(H)$, $S \in \operatorname{ker}\left(d_{A B}\right)$ implies $\left.S \in \operatorname{ker}\left(d_{A^{*} B^{*}}\right)\right)$. It is then known that the pair $(A, B)$ has the $\mathrm{PF}(d)$ property for $A, B^{*}$ belonging to a number of the commonly considered classes of operators (see [15, Theorem 3] and [8, Theorem 2]).

This paper explores the relationship between the range-kernel orthogonality of the operator $d_{A B}$ and the $\operatorname{PF}(d(S))$ property. Recall here that the element $x$ of a normed linear space $\mathscr{V}$, with norm $\|\cdot\|$, is said to be orthogonal to $y \in \mathscr{V}$ if $\|x-\lambda y\| \geq\|\lambda y\|$ 
for all complex numbers $\lambda$. Let the operator $S$ have the polar decomposition $S=$ $U|S|$; suppose that $S$ belongs to the Schatten $p$-class $\mathscr{C}_{p}$ for some $1<p<\infty$. We prove that: $\min \left\{\left\|d_{A B}(X)+S\right\|_{p},\left\|d_{A^{*} B^{*}}(X)+S\right\|_{p}\right\} \geq\|S\|_{p}$ for all $X \in \mathscr{C}_{p}$ if and only if $S \in \operatorname{ker}\left(d_{A B}\right)$ and $(A, B) \in \operatorname{PF}(d(S))$ if and only if $d_{A B}(U)=0=d_{A^{*} B^{*}}(U)$ and $\min \left\{\left\|\delta_{A}(X)+\left|S^{*}\right|\right\|_{p},\left\|\delta_{B}(X)+|S|\right\|_{p}\right\} \geq\|S\|_{p}$ for all $X \in \mathscr{C}_{p}$ (cf. [7, Theorem]). An analogue of this result is proved for the case in which $S$ is trace class and either $S$ or $S^{*}$ is injective. We also prove that if $A$ is an isometry such that $\delta_{A}(S)=0$ ( $A$ is a contraction such that $\triangle_{A}(S)=0$ ), then $\min \left\{\left\|\delta_{A}(T)+S\right\|,\left\|\delta_{A^{*}}(T)+S\right\|\right\} \geq\|S\|$ (resp., $\left.\min \left\{\left\|\triangle_{A}(T)+S\right\|,\left\|\triangle_{A^{*}}(T)+S\right\|\right\} \geq\|S\|\right)$ for all $T \in \mathscr{B}(H)$. Furthermore, if $S \in \mathscr{B}(H)$ is a smooth point, then there exists a rank one operator $X$ such that $\delta_{A}(X)=0=\delta_{A^{*}}(X)$ (resp., $\triangle_{A}(X)=0=\triangle_{A^{*}}(X)$ ). We start (see Section 2) by proving that the pair $(A, B)$ has the $\operatorname{PF}(d(S))$ property if and only if $|S|$ commutes with $B,\left|S^{*}\right|$ commutes with $A$ and $d_{A B}(U)=0$, where the partial isometry $U$ is as in the polar decomposition $S=U|S|$. This generalizes the result(s) on pairs $(A, B)$ having the $\mathrm{PF}(d)$ property and the result of Okuyama and Watanabe [14].

2. Characterizing pairs $(A, B) \in \operatorname{PF}(d(S))$. In addition to the notation already introduced, we will use the following further notation. The closure of the range of an

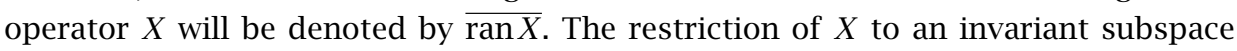
$M$ will be denoted by $\left.X\right|_{M}$, and the commutator $A B-B A$ of the operators $A, B$ will be denoted by $[A, B]$. The spectrum, the point spectrum, and the approximate point spectrum of $X$ will be denoted by $\sigma(X), \sigma_{0}(X)$, and $\sigma_{a}(X)$, respectively. The trace functional will be denoted by tr. Recall that a (completely nonunitary) contraction $A$ is said to be of the class $C_{.0}$ of contractions if $\left\|A^{* n} x\right\| \rightarrow 0$ as $n \rightarrow \infty$ for all $x \in H$. Any other notation will be explained as and when required.

The following theorem characterizes pairs of operators $(A, B)$ with the $\operatorname{PF}(d(S))$ property and is the main result of this section.

THEOREM 2.1. Let $A, B, S \in \mathscr{B}(H)$, where $S$ has the polar decomposition $S=U|S|$. Then the pair $(A, B) \in \operatorname{PF}(d(S))$ if and only if

(i) $\left[A,\left|S^{*}\right|\right]=0$;

(ii) $[B,|S|]=0$;

(iii) $d_{A B}(U)=0$.

Proof. We start by considering the case in which $d=\delta$. If $S \in \operatorname{ker}\left(\delta_{A B}\right)$ and $(A, B) \in \operatorname{PF}(\delta(S))$, then $\delta_{A B}(S)=0=\delta_{A^{*} B^{*}}(S)$, and so

$$
\begin{gathered}
A\left|S^{*}\right|^{2}=(A S) S^{*}=S\left(B S^{*}\right)=S S^{*} A=\left|S^{*}\right|^{2} A ; \\
B|S|^{2}=\left(B S^{*}\right) S=S^{*}(A S)=S^{*} S B=|S|^{2} B .
\end{gathered}
$$

This implies (i) and (ii). Also, since $\delta_{A B}(S)=0$ and $[B,|S|]=0,\left.\delta_{A B}(U)\right|_{\operatorname{ker}^{\perp} S}=0$. Clearly, $B: \operatorname{ker} S(=\operatorname{ker} U) \rightarrow \operatorname{ker} S$; hence $\delta_{A B}(U)=0$. Conversely, (ii) and (iii) together imply that $\delta_{A B}(S)=0$. Since $\overline{\text { ran } S}$ reduces $A$ (by (i)) and $\operatorname{ker}^{\perp} S$ reduces $B$ (by (ii)), it follows from $\delta_{A B}(S)=0$ that $\delta_{A_{1} B_{1}}\left(S_{1}\right)=0$, where $A_{1}=\left.A\right|_{\overline{\mathrm{ran} S}}, B_{1}=\left.B\right|_{\mathrm{ker}^{\perp} S}$ and the quasi-affinity $S_{1}: \operatorname{ker}^{\perp} S \rightarrow \overline{\operatorname{ran} S}$ is defined by setting $S_{1} x=S x$ for each $x \in \operatorname{ker}^{\perp} S$. Let $S_{1}$ have the polar decomposition $S_{1}=U_{1}\left|S_{1}\right|$; then $U_{1}$ is a unitary and $\left|S_{1}\right|$ is a quasiaffinity. Clearly, $\left[B_{1},\left|S_{1}\right|\right]=0$; hence $\delta_{A_{1} B_{1}}\left(S_{1}\right)=0$ implies that $\delta_{A_{1} B_{1}}\left(U_{1}\right)=0$, that is, 
$B_{1}=U_{1}^{*} A_{1} U_{1}$. Thus $B_{1}^{*}\left|S_{1}\right|=\left|S_{1}\right| B_{1}^{*}$ implies $U_{1}^{*} A_{1}^{*} U_{1}\left|S_{1}\right|=\left|S_{1}\right| B_{1}^{*}$, or, $\delta_{A_{1}^{*} B_{1}^{*}}\left(S_{1}\right)=0$. This implies that $\delta_{A^{*} B^{*}}(S)=0$.

Now let $d=\triangle$. If $S \in \operatorname{ker}\left(\triangle_{A B}\right)$ and $(A, B) \in \operatorname{PF}(\triangle(S))$, then $\triangle_{A^{0} B^{0}}\left(S^{0}\right)=0=$ $\triangle_{A^{0 *} B^{0 *}}\left(S^{0}\right), \overline{\operatorname{ran} S^{0}}$ reduces $A^{0}$ and $\operatorname{ker}^{\perp} S^{0}$ reduces $B^{0}$. (Here $X^{0}$ denotes the Berberian extension of the operator $X$ to a Hilbert space $H^{0} \supset H$ : recall that given a Hilbert space $H$ and an $X \in \mathscr{B}(H)$, there exists a Hilbert space $H^{0} \supset H$ and an isometric *-isomorphism $X \rightarrow X^{0}$ preserving order such that $\sigma\left(X^{0}\right)=\sigma(X), \sigma_{a}(X)=\sigma_{a}\left(X^{0}\right)=$ $\sigma_{0}\left(X^{0}\right)$ [18, page 15].) Let $A_{1}=\left.A^{0}\right|_{\overline{\operatorname{ran} S^{0}}}, B_{1}=\left.B^{0}\right|_{\operatorname{ker}^{\perp} S^{0}}$, and let $S_{1}: \operatorname{ker}^{\perp} S^{0} \rightarrow \overline{\operatorname{ran} S^{0}}$ denote the quasi-affinity defined by setting $S_{1} y=S^{0} y$ for each $y \in \operatorname{ker}^{\perp} S^{0}$. Then $\triangle_{A_{1} B_{1}}\left(S_{1}\right)=0$. As stated above, $\sigma_{a}\left(B^{0}\right)=\sigma_{0}\left(B^{0}\right)$; hence, since $S_{1}$ is a quasi-affinity, $0 \notin \sigma\left(B_{1}\right)$. We have

$$
\begin{aligned}
\triangle_{A_{1} B_{1}}\left(S_{1}\right)=0=\triangle_{A_{1}^{*} B_{1}^{*}}\left(S_{1}\right) & \Longrightarrow \delta_{A_{1} B_{1}^{-1}}\left(S_{1}\right)=0=\delta_{A_{1}^{*} B_{1}^{*-1}}\left(S_{1}\right) \\
& \Longrightarrow\left[A_{1},\left|S_{1}^{*}\right|\right]=0=\left[B_{1},\left|S_{1}\right|\right] \\
& \Longrightarrow\left[A^{0},\left|S^{0 *}\right|\right]=0=\left[B^{0},\left|S^{0}\right|\right] \\
& \Longrightarrow\left[A,\left|S^{*}\right|\right]=0=[B,|S|]
\end{aligned}
$$

where the second implication follows from the one before by the $d=\delta$ case. To prove (iii), we note that

$$
A S B=S \Longrightarrow A U|S| B=A U B|S|=\left.U|S| \Longrightarrow \triangle_{A B}(U)\right|_{\mathrm{ker}^{\perp} S}=0 .
$$

Since $B: \operatorname{ker} S \rightarrow \operatorname{ker} S$, we conclude that $\triangle_{A B}(U)=0$. To prove the sufficiency of the conditions, we note that (ii) and (iii) imply that $S \in \operatorname{ker}\left(\triangle_{A B}\right)$. As before, let $A_{1}=\left.A\right|_{\overline{\operatorname{ran} S}}$, $B_{1}=\left.B\right|_{\operatorname{ker}^{\perp} S}$ and let $S_{1}: \operatorname{ker}^{\perp} S \rightarrow \overline{\operatorname{ran} S}$ be the quasi-affinity defined by setting $S_{1} x=S x$ for each $x \in \operatorname{ker}^{\perp} S$. Then (by (i) and (ii)) $\triangle_{A_{1} B_{1}}\left(S_{1}\right)=0$, and $\left[A_{1},\left|S_{1}^{*}\right|\right]=0=\left[B_{1},\left|S_{1}\right|\right]$. Let $S_{1}$ have the polar decomposition $S_{1}=U_{1}\left|S_{1}\right| ; U_{1}$ unitary. Then $\triangle_{A_{1} B_{1}}\left(U_{1}\right)=0$, $A_{1} U_{1} B_{1}$ (in particular, $B_{1}$ ) is invertible and $A_{1}=U_{1} B_{1}^{-1} U_{1}^{*}$. We have

$$
A_{1}^{*}\left|S_{1}^{*}\right|=\left|S_{1}^{*}\right| A_{1}^{*}=\left|S_{1}^{*}\right| U_{1} B^{*-1} U_{1}^{*}=U_{1}\left|S_{1}\right| B^{*-1} U_{1}^{*} .
$$

Hence $A_{1}^{*} S_{1}=A_{1}^{*}\left|S_{1}^{*}\right| U_{1}=S_{1} B^{*-1}$, or, $\triangle_{A_{1}^{*} B_{1}^{*}}\left(S_{1}\right)=0$. This implies that $\triangle_{A^{*} B^{*}}(S)=0$, and the proof is complete.

REMARK 2.2. The hypothesis $(A, B) \in \operatorname{PF}(d(S))$ does not imply that $[A, S]=0$ (or $[B, S]=0$, or $[A,|S|]=0$, or $\left.\left[B,\left|S^{*}\right|\right]=0\right)$ for $S \in \operatorname{ker}\left(d_{A B}\right)$. Thus let $U$ be the (forward) unilateral shift and let

$$
A=U \oplus 1, \quad B=1 \oplus U^{*}, \quad S=\left[\begin{array}{cc}
0 & 0 \\
\left(1-U U^{*}\right) & 0
\end{array}\right],
$$

on $\hat{H}=H \oplus H$. Then $A, B^{*}$ are subnormal, $S \in \operatorname{ker}\left(d_{A B}\right)$ and $d_{A B}(S)=0=d_{A^{*} B^{*}}(S)$. It is easily verified that (i), (ii), and (iii) of Theorem 2.1 are satisfied, but $d_{A}(S)=-S=$ $d_{B}(S)(\neq 0)$ and $d_{A}(|S|) \neq 0 \neq d_{B}\left(\left|S^{*}\right|\right)$. The hypotheses $(A, A) \in \operatorname{PF}(d)$ and $(B, B) \in$ $\operatorname{PF}(d)$ for a class of operators $S$ in $\operatorname{ker}\left(d_{A B}\right)$ do not guarantee $(A, B) \in \operatorname{PF}(d(S))$. Thus, let $\mathscr{D}$ denote the closed unit disc in the complex plane, let $A$ be the operator of multiplication by $z$ on $\mathscr{L}^{2}(\mathscr{D})$ into itself and let $B$ be the unilateral shift (on a separable Hilbert 
space $H$ with an orthonormal basis $\left.\left\{e_{n}\right\}_{n \geq 1}\right)$. Then the only compact operator $X$ such that $\delta_{B}(X)=0$ is the zero operator (and, trivially, $(B, B) \in \operatorname{PF}\left(\left.\delta\right|_{\mathscr{K}(H)}\right)$, where $\mathscr{K}(H)$ is the ideal of compact operators). Define $S: H \rightarrow H$ by $\left(S e_{n}\right)(z)=z^{n} \chi_{\mathscr{D}_{\alpha}}$, where $\mathscr{D}_{\alpha}=$ $z:|z| \leq \alpha<1$ for some fixed $\alpha$. Then $S \in \mathscr{C}_{p}$ for all $1 \leq p<\infty$, and so is, in particular, compact. (Notice that $\operatorname{tr}\left(|S|^{2 p}\right)=\sum_{n=1}^{\infty} \int_{\mathscr{D}_{\alpha}}|z|^{2 n p} d z=2 \pi \alpha \sum_{n=1}^{\infty}\left(\alpha^{2 n p}\right) /(2 \mathrm{np}+1)<\infty$.) Clearly, $\delta_{A B}(S)=0$, but $\delta_{A^{*} B^{*}}(S) \neq 0$ : for if it were so, then we would have that $B$ has a nontrivial unitary direct summand. Again, the hypotheses $d_{A B}(S)=0=d_{A^{*} B^{*}}(S)$ do not imply that $\left.A\right|_{\overline{\text { ran } S}}$ and $\left.B\right|_{\operatorname{ker}^{\perp} S}$ are normal operators: some additional hypothesis, for example $\operatorname{ker}\left(d_{A}\right) \subseteq \operatorname{ker}\left(d_{A^{*}}\right)$ (or $\operatorname{ker}\left(d_{B}\right) \subseteq \operatorname{ker}\left(d_{B^{*}}\right)$, or more generally, $\operatorname{ker}\left(d_{A B}\right) \subseteq$ $\operatorname{ker}\left(d_{A^{*} B^{*}}\right)$ ), is required. We note here that if $\operatorname{ker}\left(d_{A}\right) \subseteq \operatorname{ker}\left(d_{A^{*}}\right)$, then $A A\left|S^{*}\right|=$ $A\left|S^{*}\right| A$ implies $A^{*} A\left|S^{*}\right|=A\left|S^{*}\right| A^{*}=A A^{*}\left|S^{*}\right|$ implies $A_{1}=\left.A\right|_{\overline{\text { ran } S}}$ is normal. Since $B_{1}\left(=\left.B\right|_{\text {err }^{\perp} S}\right)$ in the case in which $d=\delta$ and $B_{1}^{-1}$ in the case in which $d=\triangle$ is unitarily equivalent to $A_{1}$ (see the proof of Theorem 2.1), $B_{1}$ is also normal. We remark here that if $d=\triangle$, and $A, B$ are contractions (or, $d=\delta, A$ is a contraction and $B$ is invertible with $B^{-1}$ a contraction), then $(A, B) \in \operatorname{PF}\left(\left.\triangle\right|_{\mathscr{K}(H)}\right)$ (resp., $(A, B) \in \operatorname{PF} k\left(\left.\delta\right|_{\mathscr{K}(H)}\right)$ ), this follows from [5, Theorem 8 and Corollary 6.4] or [6, Theorem 2(b)].

A well-known result of Barría [3, Lemma 2] says that if $V_{1}$ and $V_{2}$ are isometries such that $\delta_{V_{1}^{*}}\left(V_{2}\right)=0$, then $\delta_{V_{1}}\left(V_{2}\right)=0$. This (indeed more) follows from our theorem, as the following argument shows. It is clear that hypotheses (ii) and (iii) of the theorem are satisfied (with $A=B=V_{1}^{*}$ and $S=V_{2}$ ). Since $V_{2}^{*} V_{1}=V_{1} V_{2}^{*}, V_{2}^{*} V_{1} V_{2}=V_{1}$, or, $V_{1}^{*} V_{2}^{*} V_{1} V_{2}=1=V_{2}^{*} V_{1}^{*} V_{2} V_{1}$. Also

$$
\begin{aligned}
\left\|\left(V_{2} V_{1} V_{2}^{*}-V_{1} V_{2} V_{2}^{*}\right) x\right\|^{2} & =2\left\|V_{2}^{*} x\right\|^{2}-2 \Re\left(V_{2}^{*} V_{1}^{*} V_{2} V_{1} V_{2}^{*} x, V_{2}^{*} x\right) \\
& =2\left\|V_{2}^{*} x\right\|^{2}-2 \mathscr{R}\left(V_{2}^{*} x, V_{2}^{*} x\right)=0
\end{aligned}
$$

for all $x \in H$. Hence, $V_{2} V_{1} V_{2}^{*}=V_{1} V_{2} V_{2}^{*}$ and $V_{2} V_{2}^{*} V_{1}=V_{2} V_{1} V_{2}^{*}=V_{1} V_{2} V_{2}^{*}$, that is, (i) of the theorem is satisfied.

Notice that $V_{2}^{*} V_{1}=V_{1} V_{2}^{*}$ implies $\triangle_{V_{2}^{*} V_{2}}\left(V_{1}\right)=0$, and the argument above shows that $\triangle_{V_{2} V_{2}^{*}}\left(V_{1}\right)=0$ also. Indeed our Theorem 2.1 generalizes a recent extension by Okuyama and Watanabe [13, Theorem] of the result of [3], as the following corollary shows.

COROLlary 2.3 (see [13, Theorem]). Let $A, B \in \mathscr{B}(H)$, and let $C$ be a partial isometry such that (i) $d_{A B}(C)=0$; (ii) $\|B\| \geq\|A\|$; (iii) $[B,|C|]=0$; and (iv) $C\left(\|B\|^{2}-B B^{*}\right)^{1 / 2}=0$. Then $d_{A^{*} B^{*}}(C)=0$.

Proof. With the partial isometry $C$ replacing the operator $S$, it is clear that hypotheses (ii) and (iii) of Theorem 2.1 are satisfied. To complete the proof we have to show that $\left[A,\left|C^{*}\right|\right]=0$.

Dividing suitably (if it needs to), we may assume that $\|B\|=1$; then $A$ is a contraction. Since $C=C\left|B^{*}\right|^{2}$ (by hypothesis (iv) above), $\delta_{A A^{*}}\left(\left|C^{*}\right|^{2}\right)=\delta_{C C^{*}}\left(\left|B^{*}\right|^{2}\right)=0$. Now extend the contraction $A$ to a partial isometry $\tilde{A}$, on $\tilde{H}=H \oplus H$ (say), by setting

$$
\widetilde{A}=\left[\begin{array}{cc}
A & \left(1-A A^{*}\right)^{1 / 2} \\
0 & 0
\end{array}\right]
$$

(see [9, page 72]), and let $X: \tilde{H} \rightarrow \tilde{H}$ be defined by $X=C C^{*} \oplus 0$. Then $\triangle_{\tilde{A} \tilde{A}^{*}}=0$, where 
$\tilde{A}$ being a partial isometry, has $C_{.0}$ completely nonunitary part. Applying [6, Theorem 2(a)], it follows that $\overline{\operatorname{ran} X}$ reduces $\widetilde{A}$ and $\left.\tilde{A}\right|_{\overline{\operatorname{ran} X}}$ is unitary. Hence $\delta_{A^{*}}(X)=0$. This implies that $\left[A,\left|C^{*}\right|^{2}\right]=0$.

Let $d_{A B}^{n}, n \geq 1$ some integer, denote an $n$-times application of $d_{A B}$. Then ker $d_{A B} \subseteq$ ker $d_{A B}^{n}$ for all $n>1$; the converse is however false in general. Additional hypotheses on $A$ and $B$, such as $A, B^{*}$ are normal or subnormal or hyponormal $[8,15]$, are required for $\operatorname{ker} d_{A B}^{n}=\operatorname{ker} d_{A B}$ to hold. An example of classes of operators $A, B^{*}$ for which ker $d_{A B}^{n}=\operatorname{ker} d_{A B}$ has been considered in [8, Lemma 4]; the following corollary generalizes [16, Theorem 1] and [8, Lemma 4]. Let $(A, B) \in \operatorname{PF}\left[d^{r}(S)\right]$, where $r$ is some natural number, denote $d_{A B}\left(d^{r}(S)\right)=0$ implies $d_{A^{*} B^{*}}\left(d^{r}(S)\right)=0$.

Corollary 2.4. Given $A, B, S \in \mathscr{B}(H)$, suppose that $(A, B) \in \operatorname{PF}\left[d^{r}(S)\right] \cap$ $\mathrm{PF}\left[A d^{r}(S)\right]$ for all $r=1,2, \ldots, n-1$. Then $d_{A B}^{n}(S)=0$ if and only if $d_{A B}(S)=0, \overline{\operatorname{ran} S}$ reduces $A$, $\operatorname{ker}^{\perp} S$ reduces $B$, and $\left.A\right|_{\overline{\operatorname{ran} S}}$ and $\left.B\right|_{\operatorname{ker}^{\perp} S}$ are normal operators.

Proof. We consider the case in which $d=\triangle$; the case $d=\delta$ is similarly dealt with. Let $S \in \operatorname{ker} \triangle_{A B}^{n}$ and let $X=\triangle_{A B}^{n-1}(S)$. The hypothesis $(A, B) \in \operatorname{PF}\left[d^{n-1}(S)\right] \cap$ PF $\left[A d^{n-1}(S)\right]$ implies that

$$
\begin{aligned}
A X B-X & =0=A^{*} X B^{*}-X \\
A(A X) B-(A X) & =0=A^{*}(A X) B^{*}-(A X),
\end{aligned}
$$

and hence that

$$
A^{*} A X B^{*}-A X=A A^{*} X B^{*}-A X \quad \text { or } \quad\left(A^{*} A-A A^{*}\right) X B^{*}=0 .
$$

Since $\overline{\operatorname{ran} X}$ reduces $A$, $\operatorname{ker}^{\perp} X$ reduces $B$, and $A_{1}=A \mid \overline{\operatorname{ran} X}$ and $B_{1}^{-1}=\left(\left.B\right|_{\operatorname{ker}^{\perp} X}\right)^{-1}$ are unitarily equivalent (see the proof of Theorem 2.1), it follows that $A_{1}$ and $B_{1}$ are normal operators. Let $S: \operatorname{ker}^{\perp} X \oplus \operatorname{ker} X \rightarrow \overline{\operatorname{ran} X} \oplus(\overline{\operatorname{ran} X})^{\perp}$ have the matrix representation $S=\left[S_{i j}\right]_{i, j=1}^{2}$. Letting $A=A_{1} \oplus A_{2}$ and $B=B_{1} \oplus B_{2}$, it then follows that

$$
\triangle_{A_{1} B_{1}}^{n}\left(S_{11}\right)=0, \quad X=\triangle_{A B}^{n-1}(S)=\left[\triangle_{A_{i} B_{j}}^{n-1}\left(S_{i j}\right)\right]_{i, j=1}^{2}=\triangle_{A_{1} B_{1}}^{n-1}\left(S_{11}\right) \oplus 0 .
$$

The operators $A_{1}$ and $B_{1}$ being normal, $\triangle_{A_{1} B_{1}}^{n}\left(S_{11}\right)=0$ if and only if $\triangle_{A_{1} B_{1}}\left(S_{11}\right)=0$; hence $X=0$. Repeating this argument a finite number of times, with $X=\triangle_{A B}^{n-1}(S)$ replaced by $X=\triangle_{A B}^{n-2}(S)$ and so forth, it now follows that $\triangle_{A B}(S)=0$, where the operators $\left.A\right|_{\overline{\text { ran } S}}$ and $\left.B\right|_{\text {ker }^{\perp} S}$ are normal.

The conclusions of Corollary 2.4 remain valid if the hypothesis that $(A, B) \in$ $\operatorname{PF}\left[d^{r}(S)\right] \cap \operatorname{PF}\left[A d^{r}(S)\right]$ is replaced by the hypothesis that $(A, B) \in \operatorname{PF}\left[d^{r}(S)\right] \cap$ $\operatorname{PF}\left[d^{r}(S) B\right]$.

REMARK 2.5. Let $\pi: \mathscr{B}(H) \rightarrow \mathscr{B}(H) / \mathscr{K}(H)$ denote the Calkin map. Let $A, B, S \in \mathscr{B}(H)$ be such that $(\pi(A), \pi(B)) \in \operatorname{PF}\left[\pi\left(d^{r}(S)\right)\right] \cap \operatorname{PF}\left[\pi\left(A d^{r}(S)\right)\right]$ for all $r=1,2, \ldots, n-1$, and $d_{A B}^{n}(S)$ is compact for some integer $n>1$. Then $\pi\left(d_{A B}^{n}(S)\right)=0$, and it follows from Corollary 2.4 that $\pi\left(d_{A B}(S)\right)=0$, that is, $d_{A B}(S)$ is compact (cf. [16, Theorem 6] and [8, Remark, page 86]). 
3. Range-kernel orthogonality and the PF-property. In this section, we explore the relationship between the range kernel orthogonality of $d_{A B}$ and the PF-property $d_{A B}(S)=0=d_{A^{*} B^{*}}(S)$. Throughout the following, we assume our Hilbert space $H$ to be separable. The operator $S$ will be said to belong to the Schatten $p$-class $\mathscr{C}_{p}=\mathscr{C}_{p}(H)$, $1 \leq p \leq \infty$, if $\|S\|_{p}=\left(\operatorname{tr}|S|^{p}\right)^{1 / p}<\infty$. The range-kernel orthogonality of $d_{A B}$, with respect to the norms $\|\cdot\|_{p}$ and $\|\cdot\|$ (= the usual operator norm), has been considered by a number of authors in the recent past (see [7, 10], and some of the references cited there). A definitive result here is the following proposition. Let $S$ have the polar decomposition $S=U|S|$.

Proposition 3.1. If $A, B \in \mathscr{B}(H)$, and $S \in \mathscr{C}_{p}$ for some $1<p<\infty$, then

$$
\left\|d_{A B}(X)+S\right\|_{p} \geq\|S\|_{p}
$$

for all $X \in \mathscr{C}_{p}$ if and only if $\operatorname{tr}\left(|S|^{p-1} U^{*} d_{A B}(X)\right)=0$ for all $X \in \mathscr{C}_{p}$ if and only if $d_{B A}\left(|S|^{p-1} U^{*}\right)=0$.

Proof. See [10, Theorem 2] and [7, Lemma 2].

Proposition 3.1 has $\|\cdot\|,\|\cdot\|_{1}$ and $\|\cdot\|_{\infty}$ analogues (see [10, Remarks, page 872] for the case $d=\delta$ ). Recall that the operator $S$ with $\|S\|=1$ is said to be a smooth point of the unit ball of $\mathscr{B}(H)$ if $\|\cdot\|$ is Gateaux differentiable at $S$, that is, if the essential norm $\|S\|_{e}$ of $S$ satisfies $\|S\|_{e}<\|S\|$, and if $S$ attains its norm at a unique (up to multiplication by a constant of modulus one) unit vector $f \in H$ [11]. (The space $\mathscr{C}_{p}$, $1<p<\infty$, being uniformly convex, every $S \in \mathscr{C}_{p}$ is a smooth point.) The following analogue of Proposition 3.1 will be required in our considerations below.

LEMMA 3.2. Let $S \in \mathscr{B}(H)$ be a smooth point, and let $f$ be the unique unit vector at which $S$ attains its norm. If $A, B \in \mathscr{B}(H)$, then the following statements are equivalent:

(i) $\left\|d_{A B}(X)+S\right\| \geq\|S\|$ for all $X \in \mathscr{B}(H)$.

(ii) $\operatorname{tr}\left((f \otimes S f) d_{A B}(X)\right)=0$ for all $X \in \mathscr{B}(H)$.

(iii) $d_{B A}(f \otimes S f)=0$.

Proof. The case $d_{A}=\delta_{A}$ is dealt with in [10, Remarks (2), page 872]; the proof of the general case follows from a similar argument (see also the proof of [7, Lemma 2]).

THEOREM 3.3. Let $S \in \mathscr{B}(H)$ be a smooth point.

(i) If $V$ is an isometry such that $\delta_{V}(S)=0$, then there exists a rank one operator $X$ such that

$$
\delta_{V}(X)=0=\delta_{V^{*}}(X) .
$$

(ii) If $A$ is a contraction such that $\triangle_{A}(S)=0$, then there exists a rank one operator $X$ such that

$$
\triangle_{A}(X)=0=\triangle_{A^{*}}(X) .
$$

The proof of the theorem proceeds through a couple of steps, stated below as lemmas. The first of these lemmas states that if $A, B$ are any contractions such that $\triangle_{A B}(T)=0$ for some $T \in \mathscr{B}(H)$, then the range of $\triangle_{A B}$ is orthogonal to $T$. This result is then used in the following lemma to prove (and extend) a result of Anderson 
[2, Theorem 1] on the range-kernel orthogonality of $\delta_{V}$ for isometries $V$. The proof of the theorem is then obtained by appealing to Lemma 3.2.

LEMMA 3.4. Let $A, B$ be contractions such that $\triangle_{A B}(S)=0$ for some $S \in \mathscr{B}(H)$. Then

$$
\left\|\triangle_{A B}(X)+S\right\| \geq\|S\|
$$

for all $X \in \mathscr{B}(H)$.

PROoF. The inspiration for the following proof comes from the proof of [2, Theorem 1].

Given $X \in \mathscr{B}(H)$, a simple calculation shows that

$$
\sum_{i=0}^{n-1} A^{n-i-1} \triangle_{A B}(X) B^{n-i-1}=A^{n} X B^{n}-X .
$$

Thus, if $S \in \operatorname{ker}\left(\triangle_{A B}\right)$, then

$$
S=-\frac{1}{n}\left\{A^{n} X B^{n}-X-\sum_{i=0}^{n-1} A^{n-i-1}\left(\triangle_{A B}(X)+S\right) B^{n-i-1}\right\} .
$$

Hence

$$
\begin{aligned}
\|S\| & \leq \frac{1}{n}\left\|A^{n} X B^{n}-X\right\|+\frac{1}{n}\left\{\sum_{i=0}^{n-1}\|A\|^{n-i-1}\|B\|^{n-i-1}\left\|\triangle_{A B}(X)+S\right\|\right\} \\
& \leq \frac{1}{n}\left\|A^{n} X B^{n}-X\right\|+\left\|\triangle_{A B}(X)+S\right\| .
\end{aligned}
$$

Letting $n \rightarrow \infty$, the proof follows.

LEMMA 3.5. Let $V$ be an isometry such that $\delta_{V}(T)=0$ for some $T \in \mathscr{B}(H)$. Then

$$
\min \left\{\left\|\delta_{V}(X)+T\right\|,\left\|\delta_{V^{*}}(X)+T\right\|\right\} \geq\|T\|
$$

for all $X \in \mathscr{B}(H)$.

Proof. If $\delta_{V}(T)=0, V$ is an isometry, then

$$
\left\|\delta_{V}(X)+T\right\| \geq\left\|V^{*}\left(\delta_{V}(X)+T\right)\right\|=\left\|-\triangle_{V^{*} V}(X)+V^{*} T\right\|=\left\|\triangle_{V^{*} V}(X)-V^{*} T\right\|
$$

for all $X \in \mathscr{B}(H)$. Since $\delta_{V}(T)=0$ implies $\triangle_{V^{*} V}(T)=0$, we have (upon choosing $A=V^{*}, B=V$ and $S=-V^{*} T$ in Lemma 3.4) that

$$
\left\|\delta_{V}(X)+T\right\| \geq\left\|\triangle_{V^{*} V}(X)+\left(-V^{*} T\right)\right\| \geq\left\|V^{*} T\right\|
$$

for all $X \in \mathscr{B}(H)$. That $\left\|\delta_{V}(X)+T\right\| \geq\|T\|$ for all $X \in \mathscr{B}(H)$ now follows from the fact that

$$
\delta_{V}(T)=0 \Longrightarrow T=V^{*} T V \Longrightarrow\|T\|=\left\|V^{*} T V\right\| \leq\left\|V^{*} T\right\|\|V\|=\left\|V^{*} T\right\| \leq\|T\| .
$$

Again, if $\delta_{V}(T)=0$ with $V$ an isometry, then

$$
\left\|\delta_{V^{*}}(X)+T\right\| \geq\left\|\left(\delta_{V^{*}}(X)+T\right) V\right\|=\left\|\triangle_{V^{*} V}(X)+T V\right\|,
$$


and hence, since $V^{*} T V-T=0$ implies $\triangle_{V^{*} V}(T V)=0$,

$$
\left\|\delta_{V^{*}}(X)+T\right\| \geq\|T V\|=\|T\|
$$

for all $X \in \mathscr{B}(H)$. This completes the proof.

Results of the type of Lemma 3.4 have been proved earlier, but under the stronger hypothesis that the intertwining operator $S$ is compact (cf. [12]). The argument of the proof of Lemma 3.5 in fact leads to a stronger result, namely that: if $A$ is left invertible by a contraction, the operator $B$ is a contraction, and if $T \in \operatorname{ker}\left(\delta_{A B}\right)$, then $\left\|\delta_{A B}(X)+T\right\| \geq\|T\|$ for all $X \in \mathscr{B}(H)$.

Proof OF THEOREM 3.3. If $V$ is an isometry such that $\delta_{V}(S)=0$, then Lemma 3.5 implies that

$$
\min \left\{\left\|\delta_{V}(X)+S\right\|,\left\|\delta_{V^{*}}(X)+S\right\|\right\} \geq\|S\|
$$

for all $X \in \mathscr{B}(H)$. Assuming now that $S$ is a smooth point, it follows from Lemma 3.2 that there exists a unique (up to multiplication by a constant of modulus one) unit vector $f \in H$ such that

$$
\delta_{V}(f \otimes S f)=0=\delta_{V^{*}}(f \otimes S f) .
$$

The operator $X=f \otimes S f$ is then the required rank one operator. Since a similar argument, using this time Lemmas 3.4 and 3.2, implies the existence of a rank one operator $X$ such that $\triangle_{A}(X)=0$, and since this (in view of the compactness of $X$ ) implies by [5, Theorem 8] that $\triangle_{A^{*}}(X)=0$, the proof is complete.

The rank one operator $X$ in Theorem 3.3 satisfies $\|X\|_{1}=\|S\|$ and $\operatorname{tr}(S X)=\|S\|^{2}$ (see [10, Lemma 1]). Also, in view of Lemma 3.2, $\delta_{V}(X)=0=\delta_{V^{*}}(X)$ if and only if $\min \left\{\left\|\delta_{V}(Y)+S\right\|,\left\|\delta_{V^{*}}(Y)+S\right\|\right\} \geq\|S\|$, and $\triangle_{A}(X)=0=\triangle_{A^{*}}(X)$ if and only if $\min \left\{\left\|\triangle_{A}(Y)+S\right\|,\left\|\triangle_{A^{*}}(Y)+S\right\|\right\} \geq\|S\|$, for all $Y \in \mathscr{B}(H)$.

We consider now the case $\left.d_{A B}\right|_{\mathscr{c}_{p}}$, where $A, B \in \mathscr{B}(H)$ and $1<p<\infty$. Recall from Proposition 3.1 that, given $S \in \mathscr{C}_{p}$,

$$
\min \left\{\left\|d_{A B}(X)+S\right\|_{p},\left\|d_{A^{*} B^{*}}(X)+S\right\|\right\} \geq\|S\|_{p}
$$

if and only if

$$
d_{B A}\left(|S|^{p-1} U^{*}\right)=0=d_{B^{*} A^{*}}\left(|S|^{p-1} U^{*}\right) .
$$

As seen in the proof of Theorem 2.1, (3.17) implies that $|S|^{2(p-1)}$ and so also $|S|$ commutes with $B,\left|S^{*}\right|=U|S| U^{*}$ commutes with $A$, and $d_{B A}\left(U^{*}\right)=0=d_{B^{*} A^{*}}\left(U^{*}\right)$. Hence $d_{B A}\left(S^{*}\right)=0=d_{B^{*}} A^{*}\left(S^{*}\right)$, that is,

$$
d_{A B}(S)=0=d_{A^{*} B^{*}}(S) .
$$

Thus, given an $S \in \mathscr{C}_{p}(1<p<\infty)$, (3.16) holds for all $X \in \mathscr{C}_{p}$ if and only if $S \in \operatorname{ker}\left(d_{A B}\right)$ and $(A, B) \in \operatorname{PF}(d(S))$ (see also [7]).

TheOREM 3.6. Let $A, B \in \mathscr{B}(H)$, and let $S(=U|S|) \in \mathscr{C}_{p}$ for some $1<p<\infty$. The following statements are equivalent:

(i) Inequality (3.16) holds for all $X \in \mathscr{C}_{p}$.

(ii) $S \in \operatorname{ker}\left(d_{A B}\right)$ and $(A, B) \in \operatorname{PF}(d(S))$. 
(iii) $d_{A B}(U)=0=d_{A^{*} B^{*}}(U)$, and

$$
\min \left\{\left\|\delta_{A}(X)+\left|S^{*}\right|\right\|_{p},\left\|\delta_{A^{*}}(X)+\left|S^{*}\right|\right\|_{p},\left\|\delta_{B}(X)+|S|\right\|_{p},\left\|\delta_{B^{*}}(X)+|S|\right\|_{p}\right\} \geq\|S\|_{p}
$$

for all $X \in \mathscr{C}_{p}$.

Proof. As seen above, (i) $\Leftrightarrow$ (ii). To prove (ii) $\Leftrightarrow($ iii), we start by noting that if (ii) holds, then, by Theorem 2.1 and its proof, $\left[A,\left|S^{*}\right|\right]=0=[B,|S|]$ and $d_{A B}(U)=0=$ $d_{A^{*} B^{*}}(U)$. Hence to prove that (ii) $\Leftrightarrow$ (iii), it will suffice to prove that inequality (3.19) holds if and only if $\left[A,\left|S^{*}\right|\right]=0=[B,|S|]$. Let $T$ denote either of $|S|$ and $\left|S^{*}\right|$. Then, since $S \in \mathscr{b}_{p}, T \in \mathscr{C}_{p}$. The map $T \rightarrow\|T\|_{p}^{p}$ is Frechèt differentiable, with the Frechèt derivative $D_{T}$ given by $D_{T}(Y)=p \Re \operatorname{tr}\left(T^{p-1} Y\right)$ (see [1, Theorem 2.1]). Let $Z=A$ or $A^{*}$ in the case in which $T=\left|S^{*}\right|$ in $\delta_{Z}(T)$, and let $Z=B$ or $B^{*}$ in the case in which $T=|S|$ in $\delta_{Z}(T)$. Then Proposition 3.1 translates to $\left\|\delta_{Z}(X)+T\right\|_{p} \geq\|T\|_{p}$ for all $X \in \mathscr{C}_{p}$ if and only if $\left(\operatorname{tr}\left(T^{p-1} \delta_{Z}(X)\right)=0\right.$ for all $X \in \mathscr{C}_{p}$ if and only if $\delta_{Z}\left(T^{p-1}\right)=0$ (see [10, Theorem 2] and [7, Lemma 2]). Hence inequality (3.19) holds if and only if $\delta_{Z}(T)=0$.

We close this paper by considering the case $\left.d_{A B}\right|_{\mathscr{C}_{1}}$. Let $S=U|S| \in \mathscr{C}_{1}$ be such that either $S$ or $S^{*}$ is injective. Then $S$ is a smooth point (of Ball $\left(\mathscr{C}_{1}\right)$ ) and the map $S \rightarrow\|S\|_{1}$ is Frechèt differentiable. Let $V=U^{*}$ if $S$ is injective and $V=U$ if $S^{*}$ is injective. Then

$$
\min \left\{\left\|d_{A B}(X)+S\right\|_{1},\left\|d_{A^{*} B^{*}}(X)+S\right\|_{1}\right\} \geq\|S\|_{1}
$$

for all $X \in \mathscr{C}_{1}$ if and only if

$$
\operatorname{tr}\left(V d_{A B}(X)\right)=0=\operatorname{tr}\left(V d_{A^{*} B^{*}}(X)\right)
$$

for all $X \in \mathscr{C}_{1}$. (This is proved for the case in which $d=\delta$ and $A=B$ in [10]; the general case follows from a similar argument.) Choose $X$ to be the rank one operator $(x \otimes y)$; $x, y \in H$.Then,since $V A X$ and $V X$ are in $\mathscr{b}_{1}$ for all $X \in \mathscr{b}_{1}$,

$$
\begin{aligned}
& \operatorname{tr}\left(V \triangle_{A B}(X)\right)=0 \Longleftrightarrow \operatorname{tr}\left(\triangle_{B A}(V) X\right)=0 \Longleftrightarrow\left(\triangle_{B A}(V) x, y\right)=0 ; \\
& \operatorname{tr}\left(V \triangle_{A^{*} B^{*}}(X)\right)=0 \Longleftrightarrow \operatorname{tr}\left(\triangle_{B^{*} A^{*}}(V) X\right)=0 \Longleftrightarrow\left(\triangle_{B^{*} A^{*}}(V) x, y\right)=0, \\
& \operatorname{tr}\left(V \delta_{A B}(X)\right)=0 \Longleftrightarrow \operatorname{tr}\left(-\delta_{B A}(V) X\right)=0 \Longleftrightarrow\left(\delta_{B A}(V) x, y\right)=0 ; \\
& \operatorname{tr}\left(V \delta_{A^{*} B^{*}}(X)\right)=0 \Longleftrightarrow \operatorname{tr}\left(-\delta_{B^{*} A^{*}}(V) X\right)=0 \Longleftrightarrow\left(\delta_{B^{*} A^{*}}(V) x, y\right)=0
\end{aligned}
$$

for all $x, y \in H$. Hence, if (3.20) holds, then

$$
d_{A B}\left(V^{*}\right)=0=d_{A^{*} B^{*}}\left(V^{*}\right) .
$$

THEOREM 3.7. Let $S \in \mathscr{C}_{1}$ be such that either $S$ or $S^{*}$ is injective. If $A, B \in \mathscr{B}(H)$ and the operator $V$ is as above, then the following statements are equivalent:

(i) Inequality (3.20) holds for all $X \in \mathscr{C}_{1}$.

(ii) $V^{*} \in \operatorname{ker}\left(d_{A B}\right)$ and $(A, B) \in \operatorname{PF}\left(d\left(V^{*}\right)\right)$.

Proof. We have already seen that (i) $\Rightarrow$ (ii). To prove that (ii) $\Rightarrow$ (i), let $X \in \mathscr{C}_{1}$. Then both $V A X$ and $V X$ are in $\mathscr{C}_{1}$. By hypothesis $d_{A B}\left(V^{*}\right)=0=d_{B A}(V)$. Hence

$$
\begin{aligned}
& \operatorname{tr}\left(V \triangle_{A B}(X)\right)=\operatorname{tr}(V A X B)-\operatorname{tr}(V X)=\operatorname{tr}(B V A X)-\operatorname{tr}(V X)=\operatorname{tr}\left(\triangle_{B A}(V) X\right)=0 \\
& \operatorname{tr}\left(V \delta_{A B}(X)\right)=\operatorname{tr}(V A X)-\operatorname{tr}(V X B)=\operatorname{tr}(V A X)-\operatorname{tr}(B V X)=\operatorname{tr}\left(-\delta_{B A}(V) X\right)=0 .
\end{aligned}
$$


Since these equalities remain true when $A$ and $B$ are replaced by $A^{*}$ and $B^{*}$, respectively, it follows (from above) that (ii) $\Rightarrow$ (i).

ACKNOWLEDGEMENT. I would like to thank the Department of Mathematics, University of Botswana, for their support during the preparation of this paper. The results of this paper were first announced at KOTAC 2000, June 22-24, held at SungKungKwan University, Suwon.

\section{REFERENCES}

[1] J. G. Aiken, J. A. Erdos, and J. A. Goldstein, Unitary approximation of positive operators, Illinois J. Math. 24 (1980), no. 1, 61-72. MR 81a:47026. Zbl 452.47025.

[2] J. Anderson, On normal derivations, Proc. Amer. Math. Soc. 38 (1973), 135-140. MR 47\#875. Zbl 255.47036.

[3] J. Barría, The commutative product $V_{1}^{*} V_{2}=V_{2} V_{1}^{*}$ for isometries $V_{1}$ and $V_{2}$, Indiana Univ. Math. J. 28 (1979), no. 4, 581-586. MR 80h:47021. Zbl 428.47019.

[4] K. N. Boyadzhiev, Commuting $C_{0}$ groups and the Fuglede-Putnam theorem, Studia Math. 81 (1985), no. 3, 303-306. MR 87b:47047. Zbl 589.47041.

[5] R. G. Douglas, On the operator equation $S^{*} X T=X$ and related topics, Acta Sci. Math. (Szeged) 30 (1969), 19-32. MR 40\#3347. Zbl 177.19204.

[6] B. P. Duggal, On intertwining operators, Monatsh. Math. 106 (1988), no. 2, 139-148. MR 89k:47031. Zbl 652.47019.

[7] _ _ Range kernel orthogonality of derivations, Linear Algebra Appl. 304 (2000), no. 13, 103-108. MR 2000k:47042. Zbl 955.47008.

[8] _ A remark on generalised Putnam-Fuglede theorems, Proc. Amer. Math. Soc. 129 (2001), no. 1, 83-87. MR 2001e:47061. Zbl 958.47015.

[9] P. R. Halmos, A Hilbert Space Problem Book, 2nd ed., Graduate Texts in Mathematics, vol. 19, Springer-Verlag, New York, 1982. MR 84e:47001. Zbl 496.47001.

[10] F. Kittaneh, Operators that are orthogonal to the range of a derivation, J. Math. Anal. Appl. 203 (1996), no. 3, 868-873. MR 97f:47033. Zbl 869.47015.

[11] F. Kittaneh and R. Younis, Smooth points of certain operator spaces, Integral Equations Operator Theory 13 (1990), no. 6, 849-855. MR 91h:47041. Zbl 738.47042.

[12] A. Mazouz, On the range and the kernel of the operator $X \mapsto A X B-X$, Proc. Amer. Math. Soc. 127 (1999), no. 7, 2105-2107. MR 99j:47051. Zbl 922.47030.

[13] R. Moore, An asymptotic Fuglede theorem, Proc. Amer. Math. Soc. 50 (1975), 138-142. MR 51\#6474. Zbl 294.47023.

[14] T. Okuyama and K. Watanabe, The Fuglede-Putnam theorem and a generalization of Barría's lemma, Proc. Amer. Math. Soc. 126 (1998), no. 9, 2631-2634. MR 98k:47034. Zbl 901.47011.

[15] M. Radjabalipour, On majorization and normality of operators, Proc. Amer. Math. Soc. 62 (1977), no. 1, 105-110. MR 55\#3856. Zbl 372.47014.

[16] Y. Tong, Kernels of generalized derivations, Acta Sci. Math. (Szeged) 54 (1990), no. 1-2, 159-169. MR 92f:47032. Zbl 731.47038.

[17] G. Weiss, The Fuglede commutativity theorem modulo the Hilbert-Schmidt class and generating functions for matrix operators. I, Trans. Amer. Math. Soc. 246 (1978), 193-209. MR 81b:47033. Zbl 403.47009.

[18] D. Xia, Spectral Theory of Hyponormal Operators, Operator Theory: Advances and Applications, vol. 10, Birkhäuser Verlag, Basel, 1983. MR 87j:47036. Zbl 523.47012.

B. P. Duggal: Department of Mathematics, Faculty of SCience, United ARAB Emirates UNIVERSity, P.O. BOX 17551, Al Ain, UNited ARAB EMIRATES

E-mail address: bpdugga1@uaeu.ac.ae 


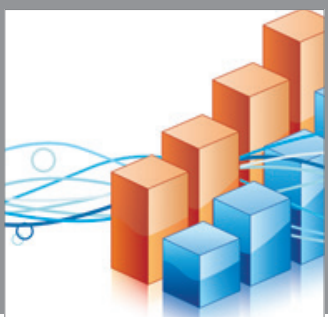

Advances in

Operations Research

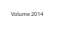

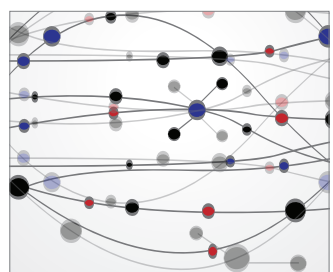

\section{The Scientific} World Journal
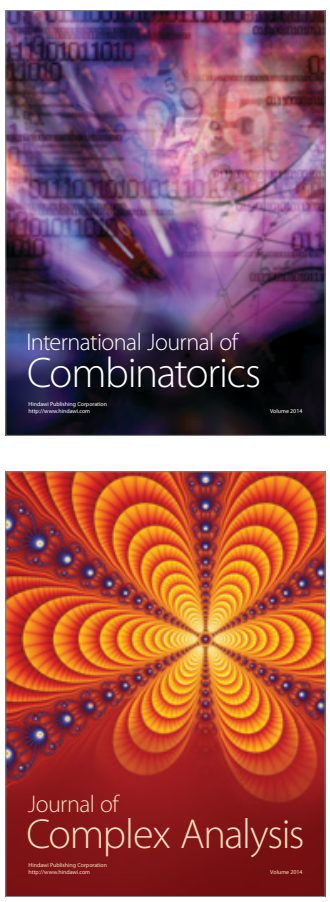

International Journal of

Mathematics and

Mathematical

Sciences
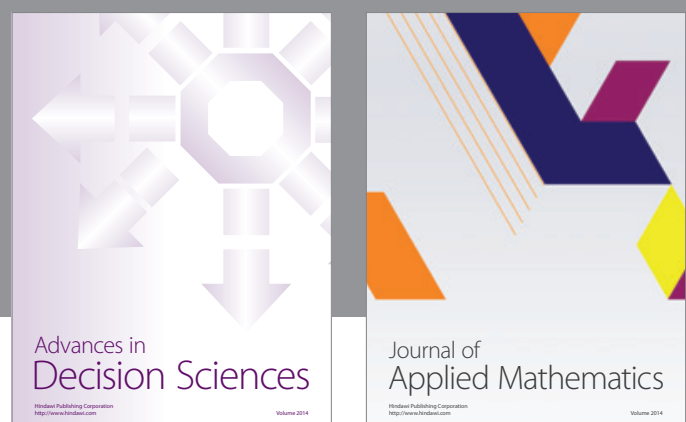

Journal of

Applied Mathematics
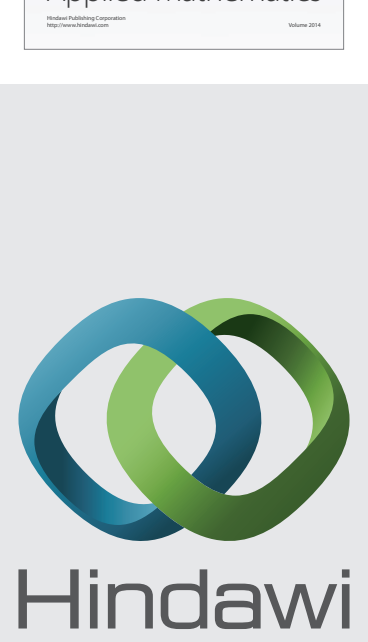

Submit your manuscripts at http://www.hindawi.com
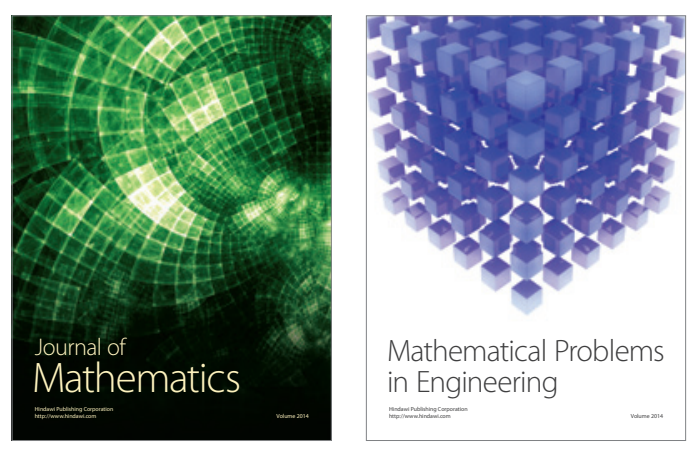

Mathematical Problems in Engineering
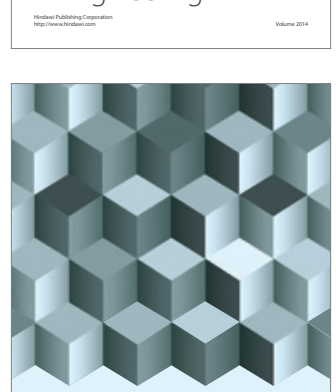

Journal of

Function Spaces
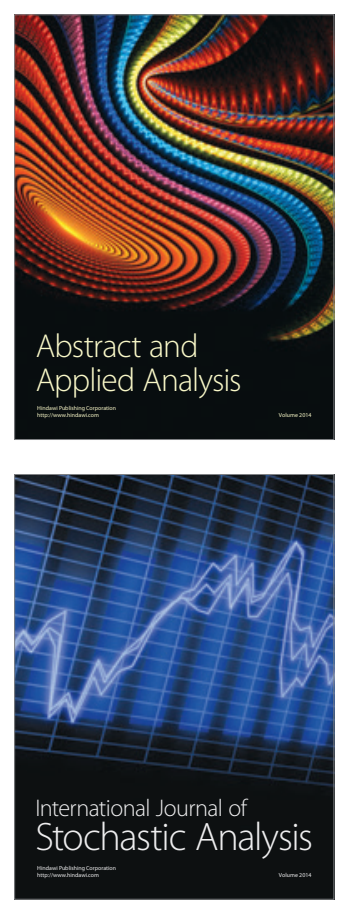

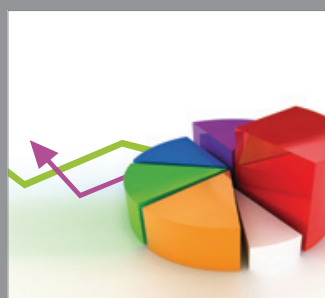

ournal of

Probability and Statistics

Promensencen
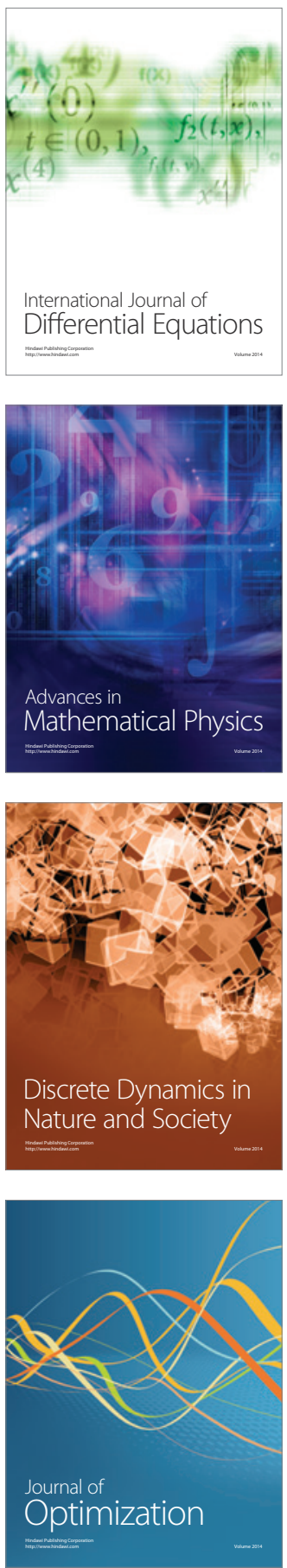